THE purpose of this article is to present some 1 measured effects of the atmosphere on sonicboom ground-pressure signatures (that is, the shapes of the pressure variation at the ground due to the boom). These data were obtained during the recent joint U. S. Air Force-National Aeronautics and Space Administration-Federal Aviation Agency (USAF-NASA-FAA) sonicboom flight-test program. ${ }^{1}$

It is well-known that sonic booms are associated with the shock waves generated by aireraft in supersonic flight. ${ }^{2}$ By way of review, the nature of the sonic-boom pressure signature at ground level can be seen in Fig. 1. The characteristic pressure
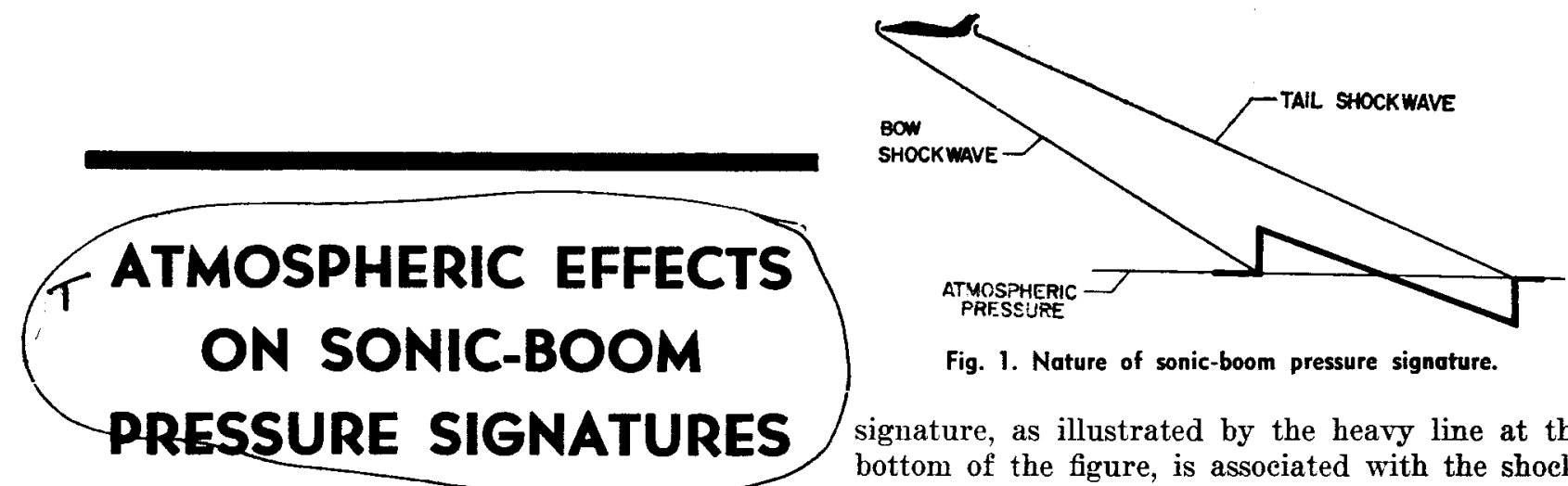

Fig. 1. Nature of sonic-boom pressure signature.

signature, as illustrated by the heavy line at the bottom of the figure, is associated with the shockwave pattern as it sweeps along the ground. Such an idealized $N$ waveform as this is hardly ever realized in practice, since it may be affected by considerations of airplane design and operation, as well as by the atmosphere (cf. Ref. 2).

Some of the atmospheric factors that may affect this wave in one way or another are pressure gradient, humidity, wind velocity and direction, windvelocity gradient, temperature gradient, and turbulence. The present tests have not been definitive enough to evaluate all of these factors, so some are discussed only briefly. The effect of temperature gradient near the ground surface, along with the associated turbulence, is considered in some detail.

Domenic J. Maglieri, an Aerospace Engineer at the NASA Langley Research Center, Langley Station, Hampton, Virginia, was graduated from the University of Pittsburgh in 1951 with a B.S. degree in mechanical engineering. He entered on duty at the NACA (currently the NASA) in 1951 and is presently assigned to the Acoustics Branch of the Dynamic Loads Division. Mr. Maglieri has been engaged in studies relating to sonic booms, jet noise, and fan and compressor noise, and has authored a number of technical papers.

Ir. Parrott is a native of Tennessee and receivcd the degree of Bachelor of Science in physics at the University of Tennessee in 1959. Since June 1959, he has been employed at the Langley Research Center of the National Aeronautics and Space Administration, Langley Station, Hampton, Virginia, where he is engaged in studies connected with jet noise and sonic boom.

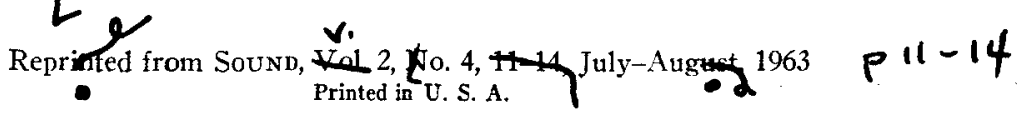

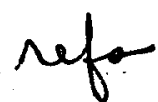

$$
\text { (NASA RP-79) }
$$




\section{Test Arrangements}

The test arrangements were similar to those reported in reference 2, but are repeated here for the sake of convenience and clarity. The area in which the tests were conducted and the arrangement of the test facilities and equipment are shown in Fig. 2 (taken from Ref. 2). Flights were made

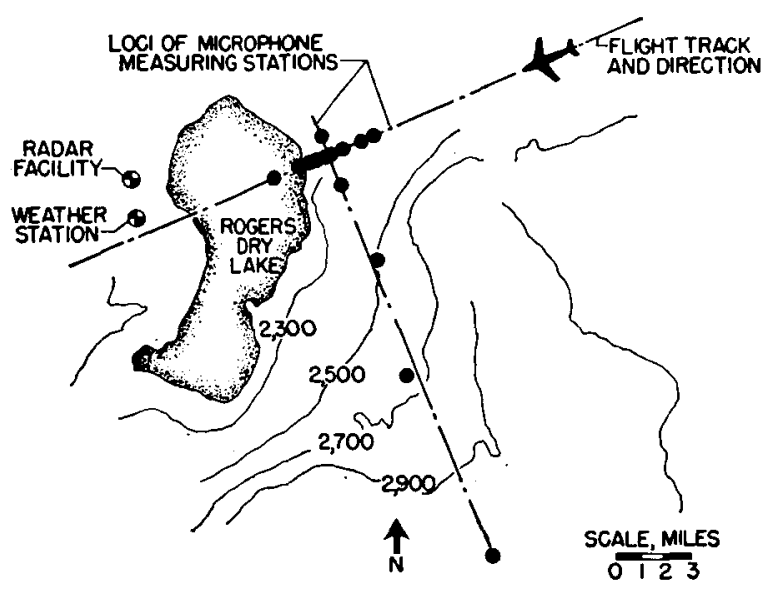

Fig. 2. Arrangement of test focilities and equipment.

in the vicinity of Edwards Air Force Base, California, during September and October of 1961. Microphones were located in the vicinity of Rogers Dry Lake and arranged in a "T"-shaped array, which had the approximate dimensions of 4 by 20 miles. The aircraft was direeted on course by means of the radar facility, and accurate tracking information was obtained during each flight. Standard rawinsonde information was obtained at $1000-\mathrm{ft}$ intervals up to the flight altitude prior to each test flight. Additional weather information for the lower atmosphere was obtained by means of wiresonde equipment at 100 -ft intervals up to about $1000 \mathrm{ft}$. Since the area was generally flat, as indicated by the contouring, it was felt that possible effects of terrain were minimized.

\section{Ground-Pressure Measurements}

The instrumentation array was used to record the ground-pressure signatures over a range of flight and atmospheric conditions along the flight track and at various lateral distances out to about 20 miles. From the measured data, some effects of the atmosphere on the pressure signatures, the peak overpressures, and the lateral-spread patterns have been determined.

\section{PRESSURE SIGNATURES}

One of the first experiments that was conducted was to group a number of microphones together to study the repeatability of measurements, where essentially the same atmospheric conditions would be expected to exist for each measurement. The results of these experiments are shown in Fig. 3 for seven microphones that were grouped within an area of about 1 square foot (sq ft). Each of the traces represents the measurement of the pressure signature at the ground surface resulting from a steady-level supersonic flight at about $41000 \mathrm{ft}$ altitude. The most obvious result is that all of these traces have about the same shape. Furthermore, the variation in the peak amplitudes was of the order of $\pm 12 \%$. These signatures differ substantially from the idealized $N$ wavesignatures shown in the sketch of Fig. 1; however, the instrumentation was adequate to have recorded an $N$ wave, had one existed. It is believed that the peculiar shape of the waves shown can be attributed largely to effects of the atmosphere; this is discussed later.

For steady flight conditions, it would be expected that the same pressure signature would exist at any point along the ground track, provided atmospheric effects were not significant. In order to obtain data of this type, the microphones were spread out along the ground track over a distance of about 2 miles and the supersonic flights were repeated. The resulting pressure signatures for two different flights are shown in Fig. 4. The data are presented for microphone locations separated by the reference distances indicated in the figure. The pressure signatures on the left were obtained during the morn-

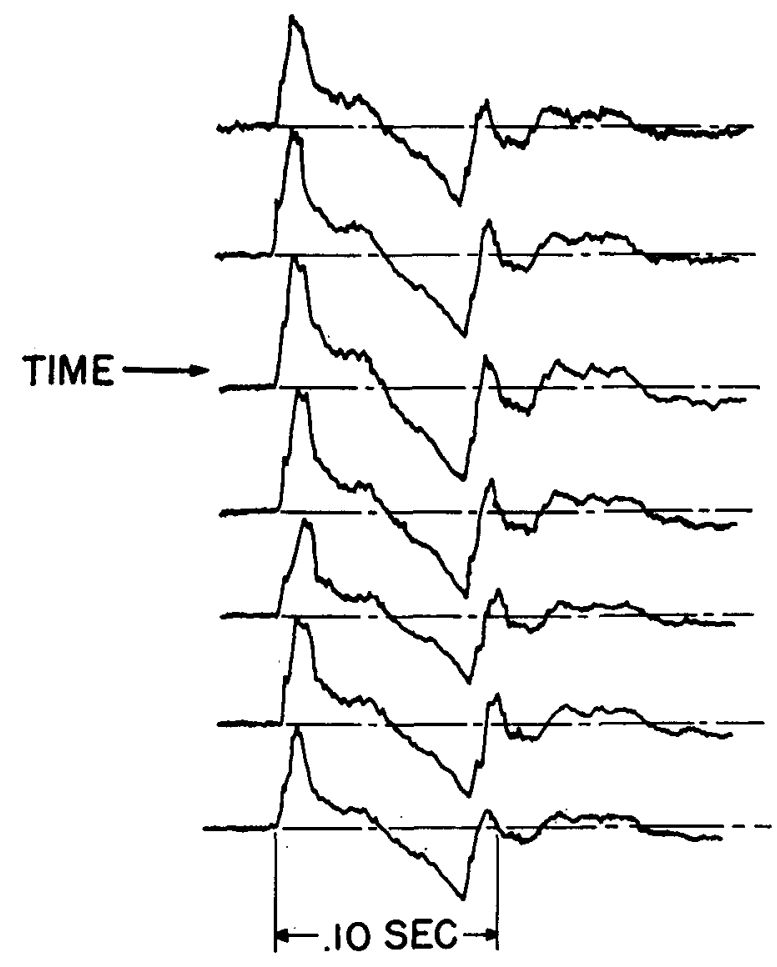

Fig. 3. Measured sonic-boom ground-pressure signatures from microphones all at one location for a fighter aircraft at Mach number 1.5 and 41000 feet altitude. 


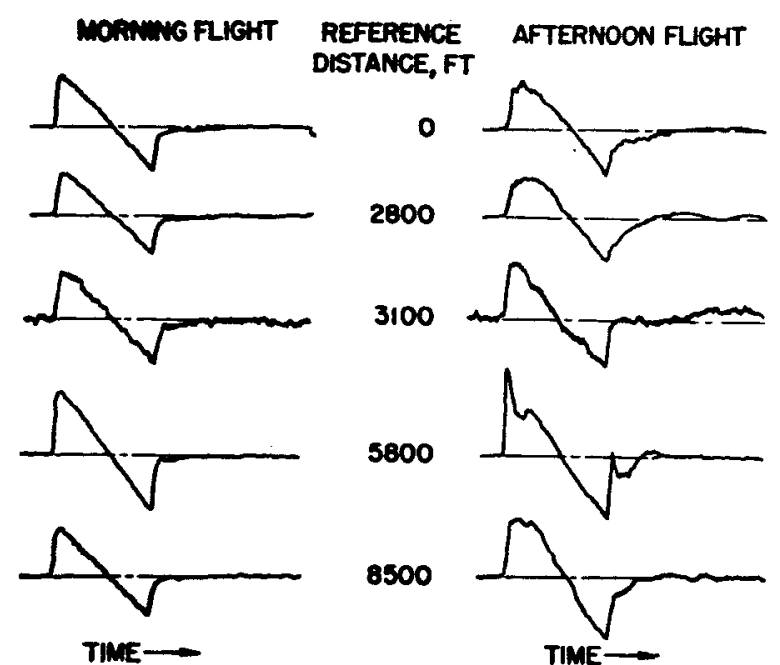

Fig. 4. Measured sonic-boom ground-pressure signatures from microphones ot axial locations along ground track of fighter aircraft at a Mach number of 1.9 and 51000 feet oltitude.

ing, whereas those on the right were obtained during the afternoon for comparable flight conditions.

It may be seen that the pressure signatures of the morning flights were consistently of the same shape even though they were measured over a large distance. Furthermore, these signatures resemble the classical $N$ waveshape. On the other hand, the waveshapes obtained during the afternoon flights at the same measuring stations differ in waveshape in an erratic manner from station to station. The most obvious difference in the atmospheric conditions for these two flights was found to be the tem. perature profile in the lower atmosphere as indicated in Fig. 5.

Figure 5 shows temperature plotted against altitude as determined from wiresonde and rawinsonde soundings taken during the times of the flights. The filled symbols represent the type of temperature profile existing for the morning flight of Fig.

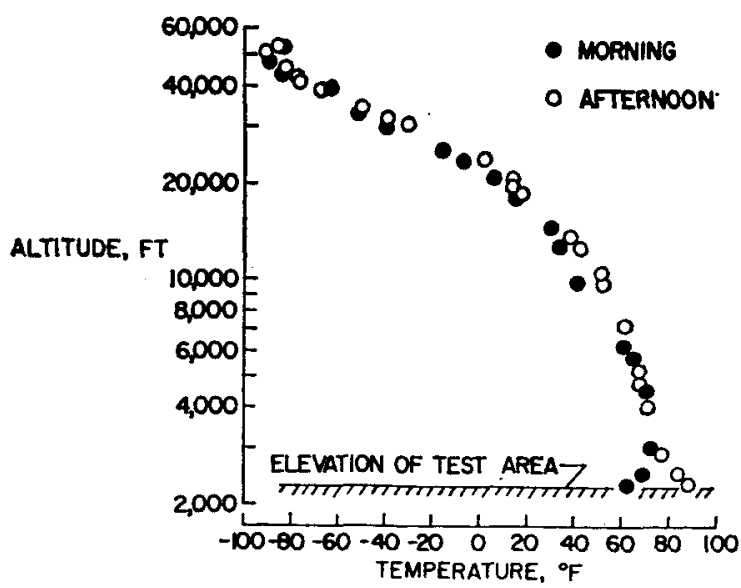

Fig. 5. Temperature profiles similar to those for the flights of Figure 4.
4 , whereas the open symbols apply to the afternoon flight. It may be seen that the temperature conditions of the upper atmosphere do not vary appreciably during the morning and afternoon. On the other hand, in the first few hundred feet of the lower atmosphere, the temperature profile varies markedly. In the morning, a temperature inversion exists during which time the surface layer of the atmosphere is quiescent. Later in the day, as the surface temperature increases, the temperature profile may change to the extent that a superadiabatic lapse-rate condition can exist as indicated. For such a temperature profile, the surface layer of the atmosphere is inherently unstable and severe thermal-induced turbulence may be generated. There is a strong correlation between the type of signature measured and the existing temperature profile in the lower atmosphere. Consistent $\boldsymbol{N}$ wave types of signatures were measured when the lower atmosphere was quiescent, whereas erratic signatures were measured when the lower atmosphere was considered to be unstable. As a matter of further information, surface winds tended to distort

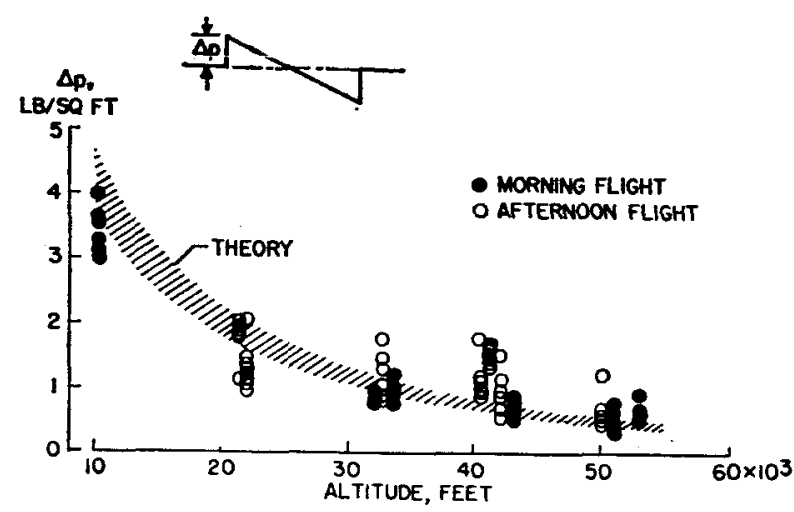

Fig. 6. Measured sonic-boom ground overpressures as a function of altitude for fighter aircraft in steady-level flight in the Mach number range 1.2-2.0.

the pressure signature, but the effects were not nearly so pronounced as those for thermal-induced turbulence.

\section{PEAK OVERPRESSURES}

Previously discussed changes in the pressure signatures also result in changes in the peak overpressures as defined in Fig. 6. As indicated in the sketch of the figure, the peak overpressure $\Delta p$ is the initial pressure jump and is an indication of the intensity of the sonic boom. These $\Delta p$ values have been measured for a large number of flights and are plotted in the figure as a function of altitude. Data are ineluded both for morning and afternoon flights as indicated by the filled and open symbols, respectively, and are compared with theoretical calculations for the range of Mach numbers 1.2-2.0 (i.e., airplane velocities $1.2-2.0$ times the velocity 
of sound). It is quite apparent from the figure that the filled symbols of the morning flights fall more nearly in line with the theory and, in general, exhibit less scatter than the afternoon flights (open symbols), which were made during the time during which much thermal activity existed in the test area.

\section{WAVEFRONTS}

In addition to the effects already discussed, the atmospheric conditions also have an effect on the speed of propagation of the shockwaves and, hence, the shape of the lateral-spread patterns. Information of this type was obtained by means of the lateral measuring stations and is presented in Fig. 7 for two flights for which the atmospheric conditions were different.

The bow shockwave from the airplane intersects the ground plane in a manner indicated by the sketch in the upper left of Fig. 7. The calculated ground-intersection curve on one side of the flight track, from an aircraft flying in a homogeneous (no wind, constant temperature) atmosphere at a Mach number of 2 and at an altitude of $50000 \mathrm{ft}$, is shown by the solid curve in the figure. It can be seen that when the airplane is in the overhead position the calculated bow-wave intersection with the ground plane is about 16 miles down the track. Accurately measured arrival-time information from stations at various distances $y$, perpendicular to the ground track, interpreted in terms of distance $x$, parallel to the ground track, are also plotted on the figure for comparison.

The filled points represent data obtained for a headwind-gradient condition for which the maximum wind velocity at altitude 50000 feet was about 50 feet per second ( $\mathrm{ft} / \mathrm{sec}$ ). The open points represent data for a tailwind gradient of about the same magnitude. It is obvious from the figure that the measured shapes of the wavefronts in the nonhomogeneous atmosphere did not differ markedly from the wavefront calculated for the homogeneous atmosphere. The measured wavefront, represented

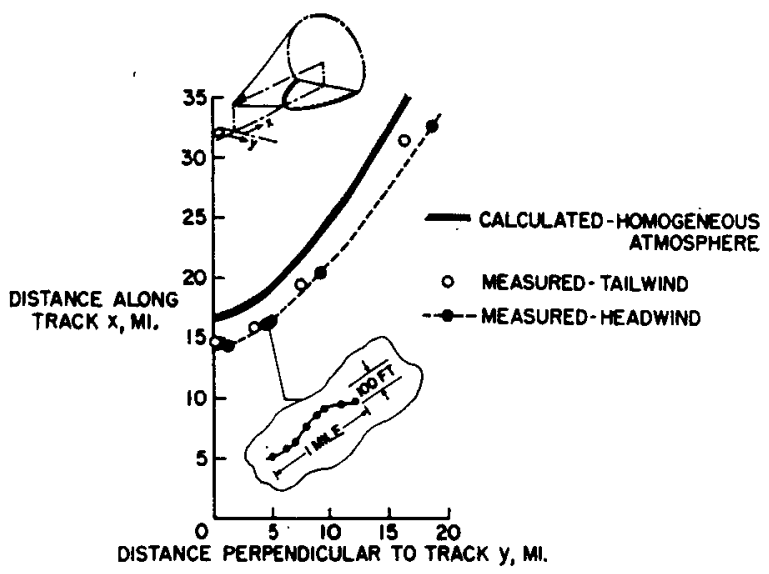

by the filled symbols, is located ahead of the wavefront represented by the open symbols. This would be expected since the wind and temperature effects are additive for a headwind condition and tend to oppose each other for a tailwind condition. The fact that both sets of data lie ahead of the calculated curve suggests that wind effects are relatively small for these tests as compared with other effects of nonhomogeneity such as temperature gradient. The differences shown are larger, however, than can be accounted for by calculations in which attempts are made to include the effects of temperature gradient.

As a matter of further information, the data of the lower sketch are presented. These data were obtained with the aid of a group of closely spaced microphones over about a 1-mile segment of the range. Local translations of the wavefront of about $100 \mathrm{ft}$ were observed over this 1-mile distance. These "ripples" in the wavefront are believed to be associated with the variations of pressure signature previously discussed.

Sonic-boom ground-pressure signatures were obtained for a wide variety of temperatures and wind profiles in the lower atmosphere. The results indicate a strong correlation between the peak overpressure and the type of signature measured, and the existing temperature profile in the lower atmosphere. Consistent $N$ wave types of signatures and peak overpressure values were measured when the lower atmosphere was quiescent, whereas relatively large variations occurred when the lower atmosphere was judged to be unstable. The gross shapes and locations of the wavefront ground-intersection patterns compare fairly well with calculations, although some local variations or ripples were observed.

1. Harvey H. Hubbard, Domenic J. Maglieri, V. Huckel, and David A. Hilton, "Ground Measurements of Sonic Boom Pressures for the Altitude Range of 10000 to 75000 Feet," NASA Tech. Mem. X-633 (1962).

2. Domenic J. Maglieri and Donald L. Lansing, "Sonic Booms from Aircraft in Maneuvers," SOUND 2, 000 (1963).

Fig. 7. Effect of the otmosphere on the intersection of the sonic-boom warefront with the ground surfoce for an oircraft in steody level flight ot a Mach number of $\mathbf{2 . 0}$ and 50000 feet altitude. 